\title{
Expression of drug transporters in the human skin: comparison in different species and models and its implication for drug development
}

\author{
Hanan Osman-Ponchet ${ }^{*}$, Alexandre Gaborit, Jean-Michel Linget and Claire E. Wilson \\ Nestlé Skin Health, les Templiers, 2400 route des Colles, BP 87, F-06902 Sophia-Antipolis

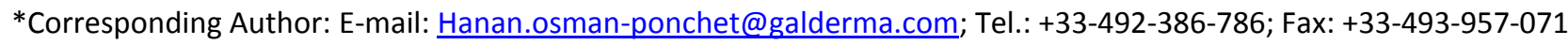 \\ Received: May 02, 2017; Revised: June 15, 2017; Published: June 22, 2017
}

\begin{abstract}
It is clear that many drug transporters (both $A B C S$ and $S L C S$ ) are present in the human skin. Different in vitro skin models can be used to investigate the role of drug transporters in the skin despite quantitative differences in expression profile across species. P-gp was shown to have an important influence on transdermal drug absorption in the skin and to function in "absorptive" transport, carrying substrate drugs from the skin surface to the dermis. This observation might be used to modulate drug distribution inside the skin. If drugs can be retained in the epidermis compartment by inhibition of the transporters, such property of the drug would be beneficial for treatment of dermatological diseases. Therefore, it might be feasible to control transdermal delivery of drugs to specific locations in the skin, by modulating the function of the transporters in the skin. We are at the dawn of an exciting period where drug transporters might be novel targets for improvement of drug delivery to the skin and for pharmacological intervention.
\end{abstract}

\section{Keywords}

Solute-carrier transporter; ATP-Binding Cassette transporter; Skin absorption

\section{Introduction}

The skin acts as an effective barrier against intrusion from the outside. The barrier may come in two ways: a physical one mainly through the stratum corneum, and a biochemical one as it has long been recognized that drug metabolizing enzymes are present in the skin: both phase I and phase II enzymes are expressed and skin metabolism has been the subject of numerous reviews [1-3] mentioning specifically the exhaustive one from Oesch et al., [4]. However, the skin is far from being a tight barrier for small lipophilic molecules as it is well known that there is a myriad of applications using the skin as the port of entry into the systemic circulation in the form of a patch, lotion, cream, ointment... [5]. The permeation through the skin of a toxic chemical may lead to serious adverse effects as the most unfortunate example of hexachlorophene poisoning in infants due to contaminated talc powder has shown [6]. So now during drug development of a new dermatological product it is mandatory to assess the uptake in the systemic circulation [7].

Normal keratinocytes express drug metabolizing enzymes $[8,9]$ but the expression of these enzymes is 
much higher in liver and intestine than in skin [10-13]. For most drugs the limiting factor for topical bioavailability is the ability to permeate through the skin and not the biochemical degradation by metabolism [14, 15]. However, it should be noted that for a compound like testosterone skin metabolism reduces sharply the topical bioavailability $[2,16]$.

Transporters are by definition vectors, as they may have an intake or efflux function. But that notion of influx or efflux is a relative one as it depends on the cell or organ we are referring to (the efflux from one organ is the intake of another one). Xenobiotic transporters are not specific, they may have several (or many) substrates and as many inhibitors (albeit with a different affinity). Drug transporters in the skin belonging to both the ATP-binding cassette (ABC) [17-23] and Solute carrier (SLC) families [17, 23-25] have been identified in man and other animal species but our knowledge of these transporters is just at the beginning and is not as advanced as for drug metabolizing enzymes.

We would like to review in the present paper our knowledge on drug transporters in the skin not by giving an exhaustive list of everything that has been done but on focusing on what is known and not known on expression and function of these transporters in the perspective of drug development. We will discuss the potential implication of the presence of these transporters on drug development both on the aspect of topical absorption and of being the target of pharmacological intervention.

\section{Skin models}

Different skin models can be used to characterize drug transporters in the skin, among them, skin organ-culture, 3D skin microtissue and 3D reconstructed epidermis models.

\section{Skin organ-culture model}

Skin organ-culture model was previously described [20, 24]. Briefly, biopsies of fresh human skin samples excised during cosmetic surgery are collected and cultivated in chemically defined skin culture medium.

\section{D human skin micro-tissue model}

3D human skin micro-tissue is a spherical in vitro skin model consisting of spherical skin equivalent (up to $500 \mu \mathrm{m}$ diameter) containing fibroblasts (dermal core-like) surrounded by keratinocytes (epidermis-like) (Figure 1A). This in vitro model provided in a 96-well format actually available as beta-testing for screening in dermatology is provided by InSphero (Switzerland).

\section{D Reconstructed human epidermis model}

Reconstructed human epidermis (RHE) consists of an air-lifted, living, multi-layered tissue construct produced in polycarbonate inserts in serum-free and chemically defined medium, featuring normal ultrastructure and functionality similar to human tissue in vivo (Figure 1B). The RHE model Episkin (France) is generated from primary normal human keratinocytes. This model most closely mimics normal skin, allowing the topical application of a great variety of products, solid, liquid, gel, cream... However, this skin model contains only one cell type, lacks skin appendages, and therefore is insufficient to adequately mimic the complexity of human skin. 
A

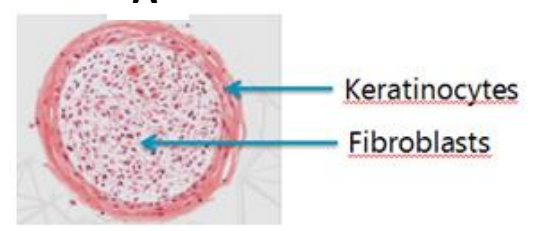

B

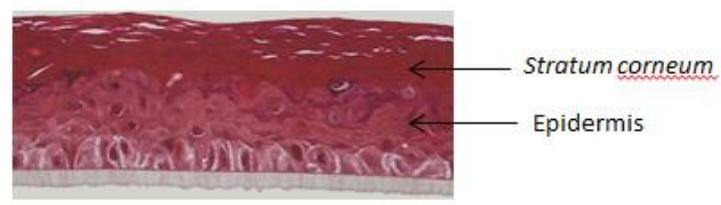

Figure 1. 3D in vitro skin models. (A) 3D human skin microtissue. (B) 3D reconstructed human epidermis.

\section{Gene expression of transporters in different species}

\section{Expression in human skin}

Several studies have reported the expression of transporters in human skin [17-25]. Their objectives and methods were not identical, but their results which cannot be exactly compared, show clearly the presence of $A B C$ and $S L C$ transporters in human skin. The semi-quantitative expression of human $A B C$ transporters in skin has been reported [21], and in this study most of the $A B C A, A B C B, A B C C, A B C D, A B C E$, $A B C F$, and $A B C G$ family members were found to be slightly (for $A B C G$ ) to moderately-highly expressed for the other family members. It should be noted that in this study a significant inter-individual variability in the expression was observed as no expression was detected in some human samples: ABCA5 and ABCF1 being the exception. In another study [20] mRNA expression of $A B C B 1, A B C C 1, A B C C 2$ and $A B C G 2$ was measured in human skin organ-culture using RT-PCR. It was found that expression of ABCC1 (MRP1) was very high compared to those of $A B C B 1, A B C C 2$, and $A B C G 2$ [20]. Comparatively to the liver or kidney the expression of $A B C$ transporters in human skin is usually lower but we found that for $A B C C 1$ the reverse is true as there is more than $\mathbf{3 0}$ fold higher expression in human skin compared to liver [20].

Many SLC and SLCO genes were found to be expressed in human skin [23-25]. One more recent study assessed the expression of SLC transporters in the human skin, liver and small intestine. While most of these transporters were moderately to highly expressed in the liver, their expression was limited in the skin and small intestine with, however, a rather high expression of SLCO2B1, SLCO4A1, SLCO16A1 and SLC16A4 in the skin [25]. In another study, of 11 transporters assessed SLC47A1 (MATE1) was found with the highest expression in the skin [24]. Comparatively with the liver it was shown that quantitatively the expression in the skin is at least 10 times lower than in the liver [24].

The study of transporters in the human skin is still in its infancy and in the 2 recent studies the expression was assessed by mRNA levels and not by the protein level nor by transporter activity. The issue of interindividual variability observed in the expression is still an open one as the number of skin samples studied so far is rather small. The normal variability has to be known before assessing for example the observation that expression of $A B C C 1$ is 26 fold lower in the skin from skin cancer patients [21].

\section{Expression in 3D human skin models}

The main $A B C$ and SLC transporters expressed in full thickness human skin in organ-culture are also expressed in both 3D human skin microtissue (Figure 2A) and 3D reconstructed human epidermis (Figure 2B). Indeed, ABCC1 (MRP1) and SLC47A1 (MATE1) are clearly expressed in those 2 models, indicating that both $3 \mathrm{D}$ in vitro skin models are suitable to evaluate drug transporters in the skin by studying their role, function, and regulation in the skin [26]. 
A

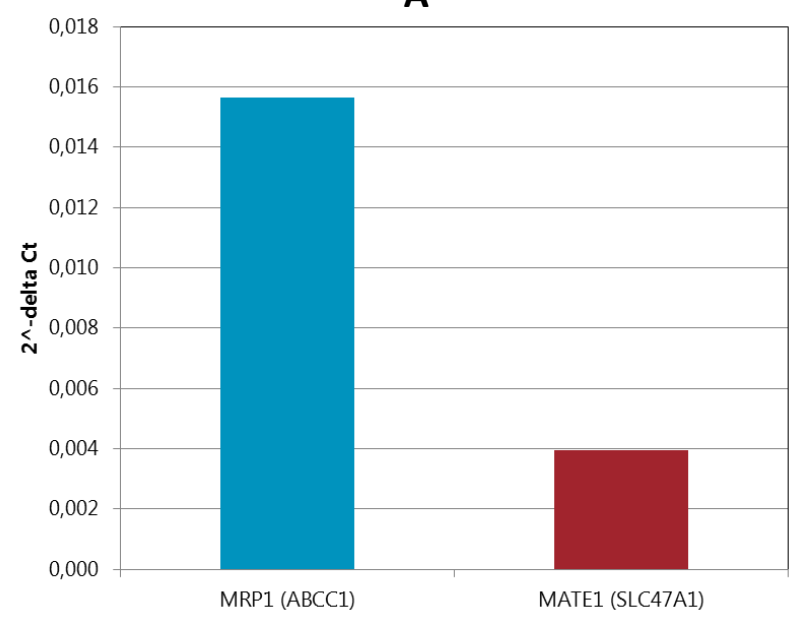

B
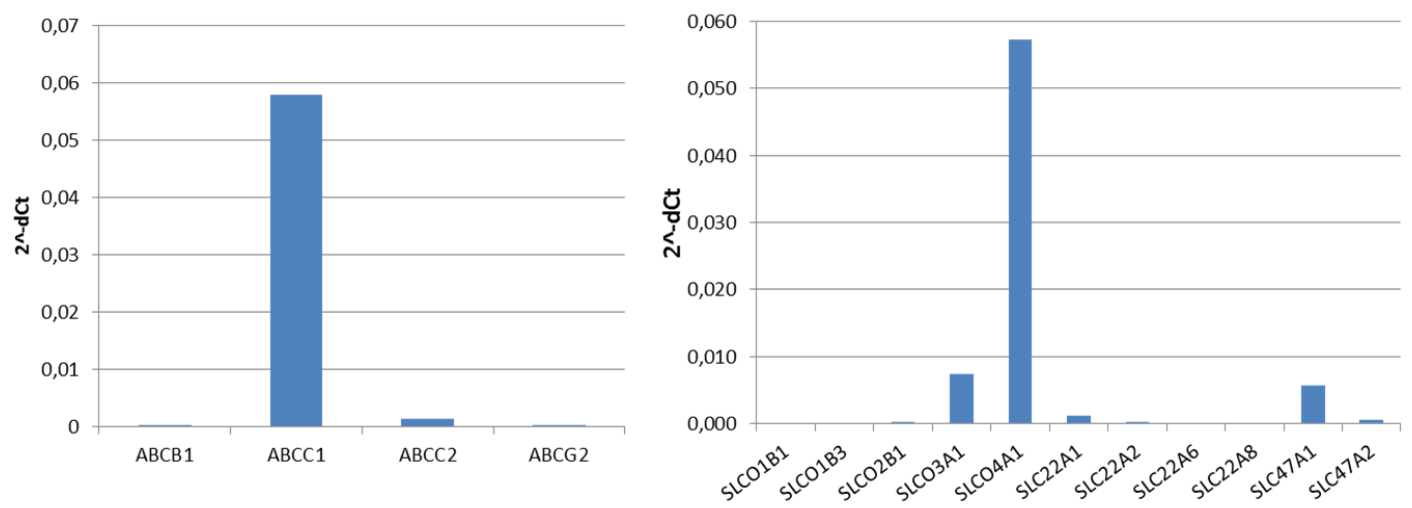

Figure 2. Constitutive expression of $A B C$ and SLC transporters in 3D in vitro human skin models. (A) 3D human skin microtissue. (B) 3D reconstructed human epidermis. Quantitative real-time reverse transcription-PCR was used to investigate the constitutive expression of $A B C$ and SLC transporters in 3D human skin models. Gene expression was normalized to 3D human skin microtissue and Reconstructed human epidermis GAPDH to ensure equality of loading. All analyses were performed in triplicate.

\section{Expression in skin from animal species}

$A B C C 1$ is the main $A B C$ transporter expressed in human skin, in Göttingen mini pig skin, and in Sprague Dawley rat skin (Figure $3 \mathrm{~A})$.

SLC47A1 is the main SLC transporter expressed in human skin and in rat skin (Figure 3B). Moreover, the expression of SLC47A1 is higher in human skin compared to rat skin. Of the four SLC transporters tested, none was expressed in mini pig skin, however, it should be noted that at present time, the gene sequence of SLC47A1 is not yet known in mini pig. Overall, these results show that expression profile of ABC and SLC transporters is qualitatively similar in skin of human and animal species tested. 
A

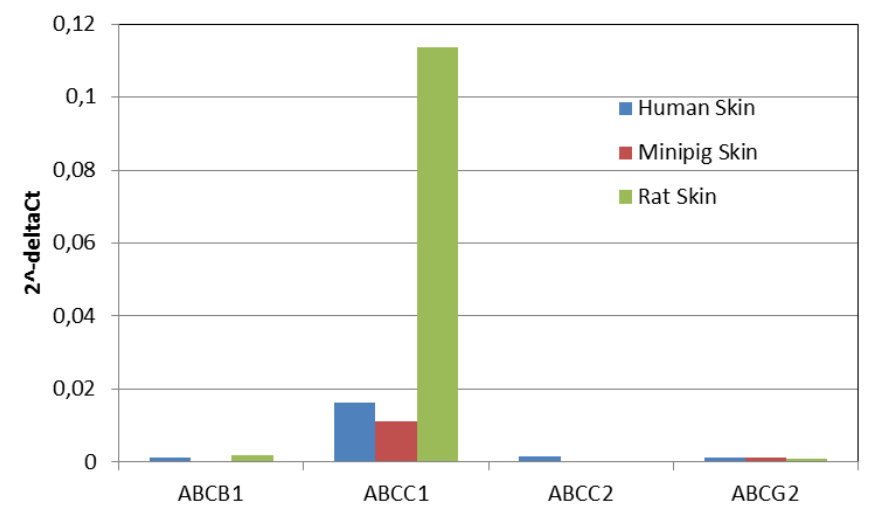

B

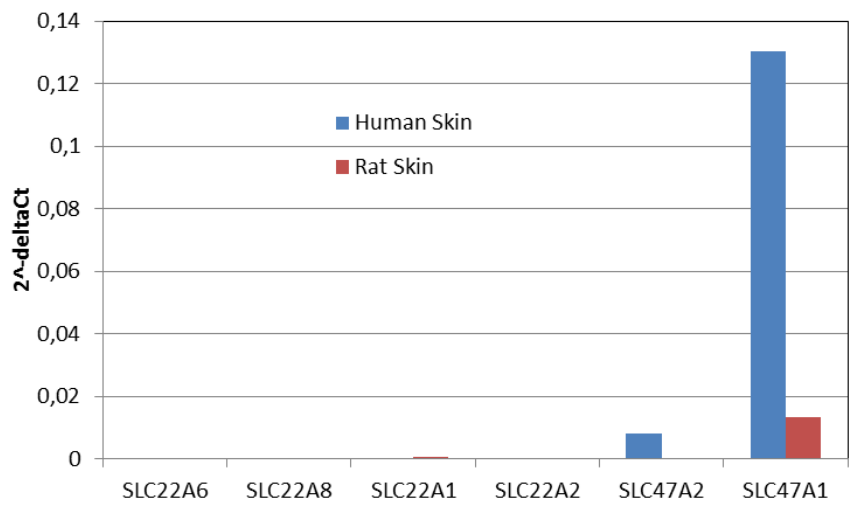

Figure 3. Constitutive expression of $A B C$ and SLC transporters in skin of different animal species. (A) Expression of $A B C$ transporters in rat, mini pig and human skin. (B) Expression of SLC transporters in rat and human skin. Quantitative real-time reverse transcription-PCR was used to investigate the constitutive expression of ABC and SLC transporters in animal skin. Gene expression was normalized to GAPDH to ensure equality of loading. All analyses were performed in triplicate.

\section{Regulation of transporter expression in the skin}

The effect of rifampicin on the expression of ABCC1 and SLC47A1, the most expressed transporters in the skin, was studied in human skin organ-culture after a 72 hour treatment period [20, 24]. Rifampicin induced a significant decrease of expression of ABCC1 [20] and SLC47A1 [24]. Expression of SLC47A2 was also decreased but not to a significant level due to the variability of expression. Interestingly UV light exposure decreased the expression of both SLC47A1 and SLC47A2, and again there is a large interindividual variability and the decrease is not significant for SLC47A2 [24].

It is difficult at this stage to draw firm conclusions as the variability in the expression blunts the results, but there is nevertheless a trend towards regulation of a few transporters in the skin. This aspect should receive more attention in the future.

\section{Localization and function of transporters in the skin}

The skin is not a homogeneous organ, so a differential expression of transporters in different skin compartments would not be unexpected. Expression of $A B C B 1$ in human skin was found to be mainly restricted to dermal components [22], sweat ducts, vessels, nerve sheaths and muscle [27], confirming an earlier observation in which P-gp in neonatal murine skin were present on the surface of basal 
keratinocytes and outer root sheath cells of hair follicles [28]. As a monoclonal antibody for human MRP1 is available we studied the localization of this transporter by immunohistochemistry, and it was found that MRP1 is mainly localized at the interface of epidermis-dermis and in the dermis in the sweat gland [20] and hair follicle (Figure 4). The concentration of expression of MRP1 in the hair follicle was confirmed in a more recent study [19]. On the other hand, BCRP was immunohistochemically identified in human epidermis and dermal endothelial cells [21] and in the hair follicle [22]. There are less data about the localization of SLC transporters in the skin; in one study SLC21A9/OATP-B was detected in the epidermis of skin specimens by immunofluorescence and immunohistochemistry [9].

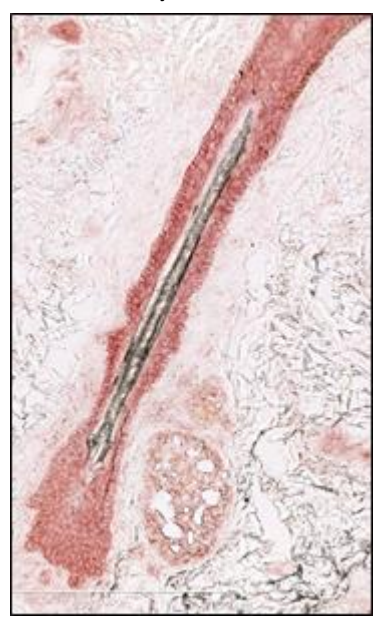

Figure 4. Localization of MRP1 in the hair follicle by immunohistochemistry.

\section{Potential function of drug transporters in the skin}

MDR1/P-gp is thought to be an efflux transporter so it was a kind of a surprise when this transporter was found to be involved in the absorptive drug transport of rhodamine 123 and itraconazole in the murine skin [18]. The penetration was lower in knockout mice (mdr1a/1b-/-) than in wild-type mice, and propranolol, a P-gp inhibitor, decreased the concentration of itraconazole in the skin of wild-type mice but not in gene knockout mice [18]. This absorptive function was confirmed with flurbiprofen and indomethacin [17]. Recently P-gp in skin was found to contribute to the transdermal absorption of topical corticoids and Ingenol-3-angelate [29, 30]. On the other hand, BCRP was shown to contribute to the transdermal transport of rhodamine 123, a substrate of P-gp/MDR1 [22].

There was still the possibility that $A B C$ transporters (other than MDR1) expressed in the skin might play a role to prevent xenobiotics from entering the systemic circulation, however, $\left[{ }^{14} \mathrm{C}\right]$-grepafloxacin was shown to be transported in mouse from the skin back to the blood [31]. In another study [20], skin absorption of rhodamine 123 and vinblastine were significantly reduced in ex vivo human skin by verapamil, an inhibitor of MRP1 [20]. Moreover, MRP1 was also found to actively reduce the accumulation of substrates within the hair follicle [19].

MDR1/P-gp, MRP1 and BCRP facilitate the flux of substrates towards the inner layers of the skin and not the reverse. So the simple concept of the skin being a barrier preventing the organ from the environment is clearly not entirely valid. The exact and final function of these transporters is still a matter of conjecture. These early studies all point out to a higher concentration of these transporters around the sweat gland and the hair follicle. The expression density of these transporters varied with the transporter being rather uniformly expressed for $A B C B 1$ to specifically expressed in the sweat gland and the dermal papilla for ABCG2 [19]. Even though the function of this differential expression has yet to be unravelled, it is 
conceivable that MRP1 provides protection against both xenobiotic and oxidative insult in the hair follicle thereby preventing melanocyte depletion and hair greying [19], and that BCRP may protect the hair follicle against cytotoxic insults from chemotherapeutics, thereby preventing or reducing the associated alopecia [19].

There are fewer studies about SLC transporters in the skin, but the uptake of large organic cations such as drugs in keratinocytes was shown to be an active process mediated by members of the SLC transporter family, which can be modulated by specific competitors in vivo [9].

Overall, the real function of transporters in the skin is still largely unknown.

\section{Implication for drug development}

Absorption through the skin is thought to be largely dependent upon the physicochemical properties of the drug and to follow the concentration gradient across the skin according the Fick's first law. This is a rough approximation as the pharmacokinetics of drug permeation is actually far more complex and should be modelized by a multicompartmental system [32].

The presence of transporters would either facilitate or hamper the topical absorption depending upon the direction of the flux. Furthermore, potential drug-drug interactions would modulate the permeation process and could either induce or inhibit the flux through a skin layer creating a local zone of abnormal concentrations. Most drugs are likely not to be affected, but in a few instances we may get a clinically significant effect. There is now evidence that substrates of P-gp have a facilitated absorption through the skin. We do not know if these theoretical considerations actually play a clinical role in the skin permeation of drugs, but in theory we could modulate the flux to either improve or decrease the systemic exposure and to increase or decrease the skin concentrations. In theory, at least, inhibitors of P-gp would reduce the systemic exposure of corticoids [29] while inducers might improve the delivery of chemotherapeutic agents into the skin.

We are at the early stage of transporter research in the skin, and we have to obtain more data about the quantitation of functional transporters and not just looking at the mRNA expression. The interindividual variability in the expression and activity of transporters is another point to clarify. Do we have genetic or disease-induced effects in the expression? So the usual drug-drug interactions regulatory guidances for other tissues (liver, intestine, kidney ...) [34, 35] may not apply until more data are obtained.

Several studies have pointed out the unusually high expression of transporters around the hair follicle and sweat gland $[19,20,22]$. The function of these transporters is not yet clear, but more research is actively needed in order to clarify the potential role in the excretory function or the protection of these organelles [19], as this may have a great potential for intervention.

Although MATE transporters belong to SLC family, they are an efflux transporter and play a role in renal and biliary excretion of endogenous and exogenous compounds including xenobiotics and drugs [36, 37]. cis-inhibition studies have suggested that MATE transporters may be involved in the transport of endogenous steroid hormones like testosterone [38, 39]. Skin is known as steroidogenic organ and skin cells produce steroid hormones like testosterone [40]. We suppose that MATE transporters are responsible for the secretion of steroid hormones through the plasma membrane in the skin. Moreover, MATE transporters are down-regulated by rifampicin which suggests a role in drug-drug interactions. Of the hypothesis that mini pig studies may better reflect human drug-induced toxicities than other species, it is mandatory to know why none of SLC transporters is expressed in the skin of Göttingen mini pig. 
One of the limitations of most of these studies is that the expression of transporters has been compared by quantifying the expression of the transporter mRNAs. This method has its shortcomings, as for transporters, the mRNA expression does not correlate well with protein expression of transporters and protein activity $[41,42]$. Quantification of protein concentration of transporter in the skin, using surrogate signature peptides and liquid chromatography-mass spectrometry, overcomes these limitations [43-45].

\section{Conclusions}

It is clear that many drug transporters (both ABCs and SLCs) are present in human skin and different in vitro skin models can be used to investigate the role of drug transporters in the skin. Despite a significant difference in expression profile across species, we are at the dawn of an exciting period where the functional aspects need to be investigated. Drug transporters were shown to have an important influence on transdermal drug absorption in the skin and to function in "absorptive" transport, carrying drugs from the skin surface to the dermis. This observation might be used to modulate drug distribution inside the skin. If drugs can be retained in the epidermis compartment by inhibition of the transporters, such property of the drug would be beneficial for treatment of dermatological diseases. Therefore, it might be feasible to control transdermal delivery of drugs to specific locations in the skin, by modulating the function of the transporters in the skin. Thus, drug transporters might be a novel target for improvement of drug delivery to the skin.

\section{References}

[1] C.K. Svensson, Drug Metabolism and Disposition, 37 (2009) 247-253.

[2] A. Mavon, C. Jacques-Jamin, Cosmetics \& Toiletries, 128 (2013) 98-103.

[3] N. Manevski, P Swart, K.K. Balavenkatratam, B. Bertschi, G. Camenish, O. Kretz, H. Schiller, M. Walles, B. Ling, R. Wettstein, D.J. Schaefer, P. Itin, J. Ashton-Chess, F. Pognan, A. Wolf, K. Litherland, Drug Metabolism and Disposition, 43 (2015) 126-139.

[4] F. Oesch, E. Fabian, K. Guth, R. Landsiedel, Archives of Toxicology, 88 (2014) 2135-2190.

[5] https://en.wikipedia.org/wiki/Topical_medication, (april 2017).

[6] G. Martin-Bouyer, M. Toga, R. Lebreton, P.D. Stolley, J. Lockhart, The Lancet, 319 (1982) 91-95.

[7] Non clinical Safety Evaluation of Reformulated Drug Products and Products for Administration by an Alternate Route, FDA Guidance for Industry and Review Staff , Oct 2015.

[8] J.M. Baron, D. Holler, R. Schiffer, S. Frankenberg, M. Neis, H.F. Merck, F.K. Jugert, Journal of Investigative Dermatology, 116 (2001) 541-548.

[9] R. Schiffer, M. Neis, D. Höller, F. Rodriguez, A. Geier, C. Gartung, F. Lammert, A. Dreuw, G. ZwadloKlarwasser, H. Merck, F. Jugert, J.M. Baron, Journal of Investigative Dermatology, 120 (2003) 285291.

[10] H. Schaeffer, T. Redelmeier, Skin barrier: Principles of percutaneous Absorption, S. Karger, Basel, CH, 1996.

[11] H. Swanson, Chemico-Biological Interactions, 149 (2004) 69-79.

[12] A. Williams, Transdermal and Topical Drug Delivery, Pharmaceutical Press, London, 2003.

[13] M. Pham, J. Magdalou, M. Totis, S. Fournel-Gigleux, G. Siest, B. Hammock, Biochemical Pharmacology, 38 (1989) 2187-2194.

[14] K. Madison, Journal of Investigative Dermatology, 121 (2003) 231-241.

[15] M. Prausnitz, S. Mitragotri, R. Langer, Nature Reviews Drug Discovery, 3 (2004) 115-124.

[16] C. Jacques, E. Perdu, E.L. Jamin, J.P. Cravedi, A. Mavon, H. Duplan, D. Zalko, Skin Pharmacol Physiol, 27 (2014) 188-200. 
[17] Q. Li, H. Tsuji, Y Kayo, Y. Sai, Y. Kubo, A. Tsuji, Journal of Controlled Release, 110 (2006) 542-546.

[18] K. Ito, H.T. Nguyen, Y. Kato, T. Wakayama, Y. Kubo, S. Iseki, A. Tsuji, Journal of Controlled Release, 131 (2008) 198-204.

[19] I.S. Haslam, C. El-Chami, A. Shahmalak, C.A. O'Neill, R. Paus, British Journal of Dermatology, 172 (2015) 1562-1572.

[20] H. Osman-Ponchet, A. Boulai, M. Kouidhi, K. Sevin, M. Alriquet, A. Gaborit, B. Bertino, P. Comby, B. Ruty, Drug Metabolism and Drug Interactions, 29 (2014) 91-100.

[21] S. Takenaka, T Itoh, R. Fujiwara, Pharmacology Research \& Perspectives, 1 (2013) 1-8.

[22] N. Hashimoto, N. Nakamichi, S. Uwafuji, K. Yoshida, T. Sugiura, A. Tsuji, Y. Kato, Journal of Controlled Release, 165 (2013) 54-61.

[23] K. Bleasby, J.C. Castle, C.J. Roberts, C. Cheng, W.J. Bailey, J.F. Sina, A.V. Kulkarni, M.J. Hafey, R.Evers, J.M. Johnson, R.G. Ulrich, J.G. Slatter, Xenobiotica, 36 (2006) 963-988.

[24] M. Alriquet, K. Sevrin, A. Gaborit, P. Comby, B. Ruty, H. Osman-Ponchet, ADMET \& DMPK, 3 (2015) 34-44.

[25] R. Fujiwara, S. Takenada, M. Hashimoto, T. Narawa, T, Itoh, Scientific Reports, 4 (2014) 1-8.

[26] H. Osman-Ponchet, 7th Euro-Global Summit on Toxicology and Applied Pharmacology, October 2426, 2016 Rome, Italy.

[27] C. Skazik, J. Wenzel, Y. Marquardt, A. Kim, H.F. Merk, D.R. Bickers; J. M. Baron, Experimental Dermatology, 20 (2011) 450-452.

[28] M.A. Sleeman, J.D. Watson, J.G. Murison, Journal of Investigative Dermatology, 115 (2000) 19-23.

[29] N. Hashimoto, N. Nakamichi, E. Yamazaki, M. Oikawa, Y. Mauo, A.H. Schinkel, Y. Kato, International Journal of Pharmaceutics, 521 (2017) 365-373.

[30] L. Li, S. Shukla, A. Lee, S.H. Garfield, D. J. Maloney, S.V. Ambudkar, S.H. Yuspa, Cancer Research, 70 (2010) 4509-4519.

[31] Q. Li, Y. Kato, Y. Sai , T. Imai, A. Tsuji, Pharmaceutical Research, 22 (2005) 842-846.

[32] Y. Dancik, O.G. Jepps, M.S. Roberts, in Dermal Absorption and Toxicity Chapter 11, University of Queensland, 2013, pp 179-207.

[33] F.G.M. Russel, Enzyme and Transporter-based drug-drug interactions. Progress and future challenges. Pang, K.S.Rodrigues, A.D. Peter, R.M. (Eds). 2010, Hardcover.

[34] Guideline on the investigation of Drug Interactions EMA, CPMP/EWP/560/95 Rev.1 June 2012.

[35] Guidance for Industry. Drug Interaction Studies. Studies design, Data Analysis, Implications for Dosing and Labeling Recommendations FDA, CDER, Feb 2012.

[36] M.H. Brown, I.T. Paulsen, R.A. Skurray, Molecular Microbiology, 31 (1999) 394-395.

[37] H. Omote, M. Hiasa, T. Matsumoto, M. Otsuka, Y. Moriyama, Trends in Pharmacological Sciences, 27 (2006) 587-593.

[38] M. Otsuka, T. Matsumoto, R. Morimoto, S. Arioka, H. Omote, Y. Moriyama, Proceedings of the National Academy of Sciences USA, 102 (2005), 17923-17928.

[39] M. Hias, T. Matsumoto, T. Komatsu, Y. Moriyama, American Journal of Physiology Cell Physiology, 291 (2006) 678-686.

[40] A. Slominski, J. Wortsman, Endocrine Reviews, 21 (2000) 457-487.

[41] S. Ohtsuki, O. Schaefer, H. Kawakami, T. Inoue, S. Liehner, A. Saito, N. Ishiguro, W. Kishimoto, E. Ludwig-Schwellinger, T. Ebner, T. Terasaki, Drug Metab Dispos, 40 (2012) 83-92.

[42] B. Prasad, R. Evers, A. Gupta, C.E. Hop, L. Salphati, S. Shukla, S.V. Ambudkar, J.D. Unadkat, Drug Metab Dispos, 42 (2014) 78-88.

[43] L. Wang, B. Prasad, L. Salphati, X. Chu, A. Gupta, C.E. Hop, R. Evers, J.D. Unadkat, Drug Metab Dispos, 43 (2015) 367-374. 
[44] B. Prasad, K. Johnson, S. Billington, C. Lee, G.W. Chung, C. D. Brown, E.J. Kelly, J. Himmelfarb, J.D. Unadkat, Drug Metab Dispos, 44 (2016) 1920-1924.

[45] H.J. Burt, A.E. Riedmaier, M.D. Harwood, H.K.Crewe, K.L. Gill, S. Neuhoff, Drug Metab Dispos, 44 (2016) 1550-1561.

(C)2017 by the authors; licensee IAPC, Zagreb, Croatia. This article is an open-access article distributed under the terms and conditions of the Creative Commons Attribution license (http://creativecommons.org/licenses/by/3.0/) (cc)) BY 